\title{
BMJ Open Associations between sitting time and non-alcoholic fatty liver diseases in Chinese male workers: a cross-sectional study
}

\author{
Huili Wei, Hua Qu, Hang Wang, Huacong Deng
}

To cite: Wei $\mathrm{H}, \mathrm{Qu} \mathrm{H}$, Wang $\mathrm{H}$, et al. Associations between sitting time and non-alcoholic fatty liver diseases in Chinese male workers: a cross-sectional study. BMJ Open 2016;6: e011939. doi:10.1136/ bmjopen-2016-011939

- Prepublication history and additional material is available. To view please visit the journal (http://dx.doi.org/ 10.1136/bmjopen-2016011939).

Received 16 March 2016 Revised 7 June 2016 Accepted 4 August 2016

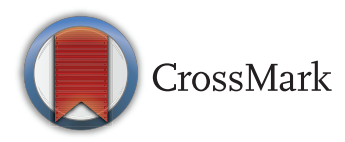

Department of Endocrinology, The First Affiliated Hospital of Chongqing Medical University, 400016 Chongqing, China, Chongqing, China

Correspondence to Professor Huacong Deng; denghuacong_111@163.com

\section{ABSTRACT}

Objectives: Various studies have revealed a close association between sedentary behaviour and metabolic diseases, yet the association between sedentary time and non-alcoholic fatty liver disease (NAFLD) remains unclear. In this study, we investigated the association between sitting time and NAFLD in a Chinese male population and explored its underlying mechanism.

Study design: A cross-sectional study.

Setting: Chongqing, China.

Participants: Our study included 2054 male participants; all of the participants were of Han nationality.

Primary outcome measures: Sitting time was assessed using a self-reported questionnaire concerning the time devoted to sitting behaviour. Various clinical and demographic biomarkers were measured. Logistic regression analyses were used to investigate the ORs and the $95 \%$ Cls between sitting time and NAFLD.

Results: We found a higher proportion of NAFLD across the tertiles of sitting time ( $p$ trend $=0.003$ ). Multivariate linear regression analyses showed sitting time independently correlated with homoeostasis model assessment for insulin resistance (HOMA-IR), alanine aminotransferase, $\gamma$-glutamyl transpeptidase, body mass index, triglyceride and the high-sensitive $C$ reactive protein (hsCRP) (all $p<0.05$ ). Further logistic regression analyses showed that longer sitting time ( $>7.1$ hours/day) was associated with a higher prevalence of NAFLD (OR 1.09; $95 \% \mathrm{Cl}$ (1.04 to 1.67)) after adjusting for confounders. However, this association was insignificant after further adjusting for hsCRP (OR 1.03; $95 \% \mathrm{Cl}$ (0.92 to 1.84)).

Conclusions: Sitting time was positively associated with the prevalence of NAFLD, and this association might be affected by inflammation.

\section{INTRODUCTION}

Non-alcoholic fatty liver disease (NAFLD) remains one of the most common chronic liver diseases worldwide; it includes a histological spectrum of diseases, such as non-
Strengths and limitations of this study

- There is little evidence on the association between sitting time and the prevalence of nonalcoholic fatty liver disease (NAFLD).

- The underlying mechanism by which sitting time contributes to the prevalence of NAFLD is unclear; inflammation may affect this association.

- Time spent on physical activity and sedentary behaviour was obtained from a self-reported questionnaire, and NAFLD was diagnosed using fatty liver index.

- The present study was a cross-sectional observation and does not imply a causal relation among sitting time, NAFLD and inflammation.

- Further research is needed to determine the chronic effects of sedentary time on NAFLD and to clarify its underlying mechanism.

alcoholic fatty liver (NAFL), non-alcoholic steatohepatitis (NASH), advanced fibrosis, cirrhosis and hepatocellular carcinoma. ${ }^{1}{ }^{2}$ Epidemiological studies revealed that the estimated prevalence rate of NAFLD may range from $5 \%$ to $40 \%$ in Asian countries and was shown to be $\sim 15 \%$ in affluent communitybased regions of China. ${ }^{3}{ }^{4}$ Several studies have shown that NAFLD was strongly related to obesity, insulin resistance, cardiovascular disease, and all-cause and liver-related mortality. ${ }^{5}$ It has been reported that NAFLD is twofold more prevalent in the overweight population and fourfold more prevalent in obese individuals. ${ }^{7}$ The prevalence of NAFLD in patients with type 2 diabetes mellitus (T2DM) is inconsistent, ranging from $\sim 45 \%$ to $75 \%$ in hospital-based studies and $30-70 \%$ in population-based studies. ${ }^{7}$ Participants with NAFLD presented with a higher risk of incidence of T2DM; NAFLD could also affect glycaemic control in patients with T2DM, indicating the vital role of NAFLD in the development and progression of diabetes. ${ }^{78}$ 
Sedentary behaviour is defined as low-energy expenditure (ranging from 1.0 to 1.5 metabolic equivalents (METs) or multiples of the basal metabolic rate) in a sitting or reclining position during waking hours. ${ }^{9}$ With advancements in modern technology, people worldwide spend more time in a sedentary lifestyle. Various studies have revealed that sedentary behaviour is a lifestyle risk factor for T2DM and obesity, and sedentary status is considered to be linked to chronic low-grade inflammation, independent of physical activity. ${ }^{10}{ }^{11}$ The American Gastroenterological Association has recommended physical activity as one of the major treatments for NAFLD. As summarised in the review by Chalasani $e t a l,{ }^{12}$ lifestyle modifications, including exercise alone or exercise combined with diet, may reduce the circulating levels of aminotransferases and improve hepatic steatosis. A retrospective analysis conducted by Kistler $e t \quad a l^{13}$ also found that vigorous-intensity physical activity was negatively associated with the severity of NAFLD.

Although a previous study revealed that physical inactivity was an important contributor to the development and progression of NAFLD, ${ }^{14}$ studies concerning the association between sedentary time and NAFLD are scarce. Evidence from Hallsworth et $a l^{15}$ showed that patients with NAFLD spent more time in sedentary behaviour, but only 74 participants had been recruited, and a study by Helajärvi et $a l^{16}$ targeted only the time spent viewing TV. Only one large sample study from Korea demonstrated a positive association between prolonged sitting time and the prevalence of NAFLD. ${ }^{17}$

Since sedentary time is strongly linked to metabolic biomarkers, whereas obesity and insulin resistance are well-established risk factors for NAFLD, targeting sedentary behaviour may provide an additional therapeutic way to improve NAFLD. This study aims to investigate the independent effects of sitting time on the metabolic risk biomarkers and NAFLD in Chinese male workers and to explore the underlying mechanism.

\section{METHODS}

\section{Ethics statement}

All of the procedures performed in this study were in accordance with the Declaration of Helsinki. Written informed consent was obtained from all of the participants.

\section{Study design and population}

We conducted a cross-sectional epidemiological study between June 2011 and December 2012 in Chongqing, China. Our study was a part of a longitudinal risk evaluation of cancer in a study of Chinese individuals with diabetes (the REACTION study). The REACTION study has been previously described in detail. ${ }^{18} \mathrm{~A}$ total of 8000 eligible participants (aged 35-75 years) were enrolled in the first year of the REACTION study in Chongqing, China, and those participants belonged to five randomly selected districts of the Chongqing municipality.
As shown in online supplementary figure $\mathrm{S} 1$, of the 8000 participants, this study excluded females $(n=4292)$ and those who did not have a job $(n=435)$. Other exclusion criteria included the following: (1) previous or present diagnoses of hepatitis B or $\mathrm{C}$ infection (including hepatitis B carrier), biliary diseases, surgical interventions $(n=147)$; (2) chronic liver diseases, such as autoimmune, Celiac and Wilson's diseases, and $\alpha$-1-antitrypsin deficiency ( $\mathrm{n}=143)$; (3) systemic illness known to cause fatty liver disease $(\mathrm{n}=44) ;(4)$ history of current or past excessive alcohol consumption, defined as average daily consumption of alcohol $>20 \mathrm{~g} /$ day $(140 \mathrm{~g} /$ week $)$ in males $(\mathrm{n}=464)$ and (5) diagnosed terminal illness prior to screening $(n=29)$ or missing values necessary for this report $(\mathrm{n}=392)$. Ultimately, this study included a total of 2054 eligible male workers (median age 56 years; IQR 51-60 years).

On the basis of the questionnaire and design of the REACTION study, data on age, education (elementary or below, junior high school, high school and university), marital status (married, divorced or single), smoking (past or current cigarette smoker), drinking (past or current drinker), total income per month $(<¥ 3000$, ¥3000- $¥ 5000$, or $>¥ 5000)$, diet, medications and family history were collected by trained investigators. All of the procedures were performed at local community-based hospitals according to the standard protocol provided by the REACTION study.

NAFLD was diagnosed based on the following criteria: (1) fatty liver index (FLI) $>60$ (sensitivity is $61 \%$; specificity is $86 \%$ ); (2) absence of previous or current diagnoses of hepatitis B or C infection, biliary diseases, autoimmune or coeliac disease and other chronic liver diseases; (3) no history of current or past excessive alcohol consumption, defined as average daily consumption of alcohol $>20 \mathrm{~g} /$ day $(140 \mathrm{~g} /$ week $)$ in males and (4) no history of systemic illness, which may cause fatty liver disease. FLI has been used to predict hepatic steatosis, and several studies have tested the sensitivity and specificity of FLI by ultrasound or liver biopsy in different populations. ${ }^{19} 20$ The diagnoses of T2DM were based on the 1999 diagnostic criteria of the WHO. ${ }^{21}$ Obesity was defined as the body mass index (BMI) $\geq 25 \mathrm{~kg} / \mathrm{m}^{2} .^{22}$

\section{Physical activity, sedentary behaviour and dietary} assessment

According to the REACTION study questionnaire, selfreported time spent on physical activity and sedentary behaviour was collected. Time spent on physical activities was measured using the frequency and average time devoted to different activities over the past month prior to the day of the interview. The METs, hour/week was assigned to all of the activities mentioned in the questionnaire, and the total MET-hour/week of each participant was computed by adding the MET-hour/week of each activity that the participant was involved. ${ }^{23}$ 
Sitting time was measured by questions, such as 'How much time did you spend sitting on weekdays and on the weekend?'. Time devoted to computer activities, watching TV or reading was also included. The weighted mean was calculated by the hours spent sitting on weekdays and the weekend.

For dietary intake, participants were asked to provide information on their regular food consumption (such as 'how much pork do you eat at a time?') and its frequency (times per day, week, month and year) as mentioned in the questionnaire.

\section{Anthropometric, clinical and demographic measurements}

For all participants, height, body weight, waist circumference, hip circumference and blood pressure (BP) were measured using standard methods. Blood samples were collected after 10 hours of food fasting and 2 hours of liquid fasting. A $75 \mathrm{~g}$ oral glucose tolerance test was conducted. Glucose was assessed using the glucose oxidase method, while glycated haemoglobin (HbAlc) was assayed using the high-performance liquid chromatography method (VARIANTTM II and D-10TM Systems; BIO-RAD, Hercules, California, USA). Fasting serum insulin (FINS) was measured using an auto-analyser (ARCHITECT i2000SR System; Abbott Laboratories, Chicago, Illinois, USA). Lipid profiles (triglyceride (TG), total cholesterol (TC), high-density lipoprotein cholesterol (HDL-C), low-density lipoprotein cholesterol (LDL-C)), high-sensitive $\mathrm{C}$ reactive protein (hsCRP), liver and kidney functions were detected by the biochemical auto-analyser (ARCHITECT c16000 System, Abbott Laboratories, Chicago, Illinois, USA). The BMI formula was computed as weight in kilograms divided by height in metres squared. The waist-to-hip ratio (WHR) formula was waist circumference in centimetres divided by hip circumference in centimetres. The homoeostasis model assessment for insulin resistance (HOMA-IR) was computed as follows: FINS $(\mathrm{mU} / \mathrm{L}) \times$ fasting plasma glucose (FPG) $(\mathrm{mmol} / \mathrm{L}) / 22.5$. FLI was calculated as follows: $\left(\mathrm{e}^{0.953 \times \ln \text { (triglycerides, } \mathrm{mg} / \mathrm{dL})}+0.139 \times \mathrm{BMI}\left(\mathrm{kg} / \mathrm{m}^{2}\right)\right.$ $+0.718 \times \ln ($ ggt, $\mathrm{U} / \mathrm{L})+0.053 \times$ waist circumference $(\mathrm{cm})$

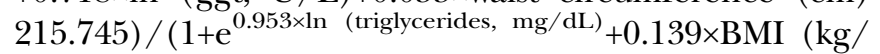
$\left.\mathrm{m}^{2}\right)+0.718 \times \ln \quad(\mathrm{ggt}, \quad \mathrm{U} / \mathrm{L})+0.053 \times$ waist circumference (cm) 215.745) $\times 100$.

\section{Statistical analysis}

SPSS software (IBM, Armonk, New York, USA V.19.0) was used for all of the statistical analyses in our study. Data were presented as the means $\pm \mathrm{SD}$ for normally distributed parameters, while median (IQR 25-75\%) was performed for non-normally distributed continuous variables, and proportions were used for categorical variables. For further analyses, non-normally distributed parameters such as TG, alanine aminotransferase (ALAT), aspartate aminotransferase (ASAT), creatinine $(\mathrm{Cr})$, FLI and total energy intake and its components were logarithmically transformed to approximate a normal distribution. Differences between multiple groups were tested by analysis of variance (ANOVA) for continuous variables, and a $\chi^{2}$ test was used to test the differences in the distribution of categorical variables. A linear-by-linear association of the $\chi^{2}$ test was used for the trend test. Partial correlation analyses were used to determine the interrelationships between variables. A multivariate linear regression was performed to identify the independent risk factors of sitting time. The multivariate logistic regression models were used to analyse the ORs and 95\% CIs between the tertiles of sitting time and NAFLD. Sitting time in tertile 1 was used as the reference group. Covariates included age, marital status, education, smoking and drinking history, monthly income, type of work, systolic BP (SBP), diastolic BP (DBP), MET (hour/week), TC, LDL-C, HDL-C, HOMA-IR, diabetes and hsCRP. For all of the statistical tests, $p$ values $<0.05$ were considered significant.

\section{RESULTS}

\section{The characteristics of participants}

As shown in table 1, participants were classified into three groups according to the tertiles of self-reported sitting time (2.2-5.0, 5.0-6.9 and >7.1 hour/day). The most sedentary group had the highest levels of ALAT, ASAT, $\gamma$-glutamyl transpeptidase (GGT), BMI, WHR, FPG, 2 hour postchallenge plasma glucose (2-hPG), HbA1c, HOMA-IR, TG, TC and LDL-C, and the lowest levels of MET and HDL-C (all parameters $\mathrm{p}<0.05$ ). The levels of the inflammatory indicator, hsCRP, were significantly higher in the most sedentary group $(p<0.05)$. A greater number of participants in the longer sitting group attended college and had office work compared with the least sedentary group. There were no significant differences in terms of age, SBP, DBP, serum Cr, alcohol, smoking, marital status and total calorie intake (all $\mathrm{p}>0.05$ ).

Furthermore, we found higher proportions of NAFLD $(6.4 \%, 7.8 \%, 11.1 \%$; p for trend=0.003), T2DM $(7.4 \%$, $8.7 \%, 12.5 \%$; p for trend $<0.001)$ and obesity $(3.2 \%$, $6.1 \%, 9.4 \%$; $\mathrm{p}$ for trend $=0.029$ ) across the tertiles of sitting time (table 2).

\section{Correlations among sitting time and other parameters}

Partial correlation analyses controlling for age showed that sitting time was positively associated with circulating levels of ALAT, ASAT, GGT, BMI, WHR, FPG, 2-hPG, HbA1c, HOMA-IR, TG, LDL-C and hsCRP, while it was negatively associated with HDL-C (table 3 , all parameters $\mathrm{p}<0.05)$. Further multivariate linear regression analyses showed that sitting time independently correlated with HOMA-IR $(\beta=0.442, p<0.001)$, ALAT $(\beta=0.186, p=0.019)$, GGT $(\beta=0.529, p<0.001)$, BMI $(\beta=0.174, p=0.022)$, TG $(\beta=0.104, p=0.041)$ and hsCRP $(\beta=0.137, p=0.032)$.

\section{Sitting time, inflammation and NAFLD}

To explore the effects of inflammation on the relationship between sitting time and NAFLD, multivariate 
Table 1 Characteristics of the participants by tertiles of sitting time

\begin{tabular}{|c|c|c|c|c|c|}
\hline $\begin{array}{l}\text { Duration of sitting time } \\
\text { (hours/day) }\end{array}$ & Tertile $1(2.2-5.0 \mathrm{~h})$ & Tertile $2(5.0-6.9 \mathrm{~h})$ & Tertile $3(>7.1 \mathrm{~h})$ & $F / \chi^{2}$ & p Value \\
\hline Participants (number) & 677 & 695 & 682 & & \\
\hline Sitting time (hours/day) & $4.4 \pm 0.6$ & $5.9 \pm 0.5^{\star}$ & $7.7 \pm 0.8^{*} \dagger$ & 9.32 & $<0.001$ \\
\hline Age (years) & $57.7 \pm 6.6$ & $57.3 \pm 6.0$ & $56.8 \pm 5.5$ & 2.384 & 0.093 \\
\hline BMI $\left(\mathrm{kg} / \mathrm{m}^{2}\right)$ & $22.92 \pm 1.94$ & $22.79 \pm 2.03$ & $23.13 \pm 2.08^{\star} \dagger$ & 4.027 & 0.024 \\
\hline WHR & $0.85 \pm 0.05$ & $0.87 \pm 0.05$ & $0.89 \pm 0.09^{\star} \dagger$ & 3.99 & 0.03 \\
\hline SBP $(\mathrm{mm} \mathrm{Hg})$ & $124 \pm 16$ & $123 \pm 16$ & $124 \pm 15$ & 0.227 & 0.797 \\
\hline $\mathrm{DBP}(\mathrm{mm} \mathrm{Hg})$ & $76 \pm 7.9$ & $76 \pm 8.0$ & $77 \pm 8.0$ & 0.562 & 0.57 \\
\hline $\mathrm{TC}(\mathrm{mmol} / \mathrm{L})$ & $4.28 \pm 0.76$ & $4.53 \pm 0.79^{*}$ & $4.84 \pm 0.84^{*} \dagger$ & 2.69 & 0.043 \\
\hline HDL-C (mmol/L) & $1.31 \pm 0.17$ & $1.29 \pm 0.19$ & $1.25 \pm 0.24^{*} \dagger$ & 9.517 & $<0.001$ \\
\hline LDL-C (mmol/L) & $2.57 \pm 0.39$ & $2.64 \pm 0.43^{*}$ & $2.77 \pm 0.43^{\star} \dagger$ & 14.01 & $<0.001$ \\
\hline $\mathrm{TG}(\mathrm{mmol} / \mathrm{L}) \ddagger$ & $1.04(0.81-1.35)$ & $1.39(1.13-1.71)^{\star}$ & $1.58(1.10-1.86)^{\star} \dagger$ & 9.22 & $<0.001$ \\
\hline $\mathrm{Cr}(\mu \mathrm{mol} / \mathrm{L}) \ddagger$ & $58(52-68)$ & $61(55-70)$ & $59(54-72)$ & 0.383 & 0.227 \\
\hline ALATT (U/L)‡ & $16(13-23)$ & $18(14-25)^{\star}$ & $20(15-27)^{\star} \dagger$ & 7.12 & 0.001 \\
\hline ASAT (U/L)‡ & $19(15-25)$ & $21(17-28)$ & $23(17-30)^{\star} \dagger$ & 3.35 & 0.029 \\
\hline ASAT/ALAT $\ddagger$ & $1.13(0.96-1.36)$ & $1.14(1.05-1.29)$ & $1.13(1.04-1.20)$ & 2.29 & 0.133 \\
\hline GGT (U/L) & $22.0 \pm 7.4$ & $23.0 \pm 8.7^{\star}$ & $24.0 \pm 9.6^{*} \dagger$ & 9.52 & $<0.001$ \\
\hline $\mathrm{FPG}(\mathrm{mmol} / \mathrm{L})$ & $5.6 \pm 0.5$ & $5.7 \pm 0.9$ & $6.0 \pm 0.9^{*} \dagger$ & 7.231 & 0.001 \\
\hline 2-hPG (mmol/L) & $6.8 \pm 2.5$ & $6.9 \pm 1.7^{\star}$ & $7.4 \pm 2.0^{*} \dagger$ & 6.028 & 0.004 \\
\hline $\mathrm{HbA} 1 \mathrm{c}(\%)$ & $5.4 \pm 0.6$ & $5.5 \pm 0.7$ & $5.7 \pm 0.8^{*} \dagger$ & 7.93 & $<0.001$ \\
\hline FINS (mU/mL) & $5.5 \pm 1.0$ & $5.6 \pm 0.8^{*}$ & $6.0 \pm 1.2$ & 1.02 & 0.067 \\
\hline HOMA-IR & $1.41 \pm 0.36$ & $1.43 \pm 0.35$ & $1.58 \pm 0.39^{*} \dagger$ & 13.09 & $<0.001$ \\
\hline MET (hours/week) & $44.7 \pm 14.3$ & $38.0 \pm 11.7$ & $26.7 \pm 12.4^{*} \dagger$ & 2.59 & 0.041 \\
\hline $\mathrm{hsCRP}(\mathrm{mg} / \mathrm{L})$ & $0.79 \pm 0.14$ & $0.92 \pm 0.17$ & $1.13 \pm 0.23$ & 2.718 & 0.011 \\
\hline FLI¥ & $16.7(6.9-33.2)$ & $18.6(7.5-36.8)^{\star}$ & $23.1(8.2-39.3)^{\star} \dagger$ & 3.58 & 0.017 \\
\hline Total energy (kcal/day)‡ & 2783.7 (2572.6-2912.4) & $2812.5(2541.9-3139.7)$ & $2817.3(2552.6-3228.1)$ & 1.93 & 0.051 \\
\hline Carbohydrates (\%)‡ & $47.2(32.8-54.1)$ & $46.8(34.7-57.2)$ & $47.1(34.6-59.3)$ & 0.72 & 0.249 \\
\hline Proteins (\%)‡ & $15.9(14.8-16.6)$ & $16.3(14.2-17.5)$ & $16.1(14.1-17.3)$ & 0.34 & 0.177 \\
\hline Lipids (\%)‡ & $36.9(28.9-44.1)$ & $36.9(28.9-43.7)$ & $36.8(28.3-44.5)$ & 0.432 & 0.098 \\
\hline Manual work (\%) & 70.8 & 66.1 & 61.3 & 2.131 & 0.043 \\
\hline Office work (\%) & 29.2 & 33.9 & 38.7 & 3.295 & 0.024 \\
\hline Past smokers (\%) & 36.1 & 30.2 & 32.6 & 0.871 & 0.929 \\
\hline Current smokers (\%) & 63.9 & 69.8 & 67.4 & 0.724 & 0.833 \\
\hline Past drinkers (\%) & 47.7 & 46.8 & 43.6 & 2.98 & 0.056 \\
\hline Current drinkers (\%) & 52.3 & 53.2 & 56.4 & 1.79 & 0.271 \\
\hline Married (\%) & 84.1 & 87.8 & 80.1 & 2.452 & 0.874 \\
\hline Divorced (\%) & 3.6 & 3.1 & 2.8 & 1.926 & 0.375 \\
\hline Single $(\%) \S$ & 12.3 & 9.2 & 17 & 0.947 & 0.826 \\
\hline Level of education (\%) & - & - & - & 2.29 & 0.042 \\
\hline Elementary or below & 46.6 & 50.5 & 47.5 & & \\
\hline Junior high school & 37.2 & 32.2 & 31.6 & & \\
\hline High school & 13.7 & 13.6 & 16.7 & & \\
\hline University & 2.5 & 3.7 & 4.3 & & \\
\hline Income (yuan/month) & - & - & - & 1.15 & 0.215 \\
\hline$<3000(\%)$ & 34.3 & 31.9 & 28.1 & & \\
\hline $3000-5000$ (\%) & 22 & 24.1 & 24.1 & & \\
\hline$>5000(\%)$ & 43.7 & 44.1 & 47.7 & & \\
\hline
\end{tabular}

Data were expressed as means \pm SD for normally distributed parameters, median (IQR 25-75\%) for abnormally distributed parameters and proportions for categorical variables. For intergroup comparison analysis, non-normally distributed parameters (including TG, ALAT, ASAT, Cr, $\mathrm{AFI}$, total energy intake and its components) were logarithmically transformed. Differences between multiple groups were tested by ANOVA for continuous variables, and $\chi^{2}$ test was used to test the differences in the distribution of categorical variables.

${ }^{*} p<0.05$ compared with tertile $1(2.2-5.0 \mathrm{~h})$.

$\mathrm{tp}<0.05$ compared with tertile $2(2.2-5.0 \mathrm{~h})$.

‡Non-normally distributed parameter.

$\S$ 'Single people' include male participants who are unmarried or windowed.

2-hPG, 2 hour postchallenge plasma glucose; ALAT, alanine aminotransferase; ANOVA, analysis of variance; ASAT, aspartate

aminotransferase; BMI, body mass index; $\mathrm{Cr}$, creatinine; DBP, diastolic blood pressure; $\mathrm{F} / \chi^{2}$, differences between groups were tested using ANOVA for continuous variables and the $\chi^{2}$ test for categorical variables; FINS, fasting serum insulin; FLI, fatty liver index; FPG, fasting plasma glucose; GGT, $\gamma$-glutamyl transpeptidase; HbA1c, glycated haemoglobin; HDL-C, high-density lipoprotein cholesterol; HOMA-IR, homoeostasis model assessment for insulin resistance; hsCRP, high-sensitive $C$ reactive protein; LDL-C, low-density lipoprotein cholesterol; MET, metabolic equivalent; SBP, systolic blood pressure; TC, total cholesterol; TG, triglyceride; WHR, waist-to-hip ratio. 
Table 2 Prevalence of NAFLD, T2DM and obesity according to the tertiles of sitting time (hour/day)

\section{Tertiles of sitting time} (hours/day)

\begin{tabular}{lllllr} 
& $\mathbf{2 . 2 - 5 . 0}$ & $\mathbf{5 . 0 - 6 . 9}$ & $\mathbf{7 7 . 1}$ & $\chi^{2}$ & p trend \\
\hline T2DM & $50(7.4 \%)$ & $60(8.7 \%)$ & $85(12.5 \%)$ & 7.735 & $<0.001$ \\
Obesity & $21(3.2 \%)$ & $42(6.1 \%)$ & $64(9.4 \%)$ & 3.107 & 0.029 \\
NAFLD & $43(6.4 \%)$ & $54(7.8 \%)$ & $75(11.1 \%)$ & 5.119 & 0.003 \\
\hline
\end{tabular}

Data were presented as proportions (\%). NAFLD was diagnosed using the FLI. $\chi^{2}, \chi^{2}$ test was used to compare the difference among groups. $p$ trend, linear-by-linear association of $\chi^{2}$ test was used for trend test.

FLI, fatty liver index; NAFLD, non-alcoholic fatty liver disease; T2DM, type 2 diabetes mellitus.

Table 3 Age-adjusted correlation analysis between sitting time and other parameters

\begin{tabular}{lrr}
\hline Variables & \multicolumn{1}{r}{$r$} & $p$ Value \\
\hline FPG $(\mathrm{mmol} / \mathrm{L})$ & 0.302 & $<0.001$ \\
2-hPG $(\mathrm{mmol} / \mathrm{L})$ & 0.243 & 0.006 \\
HbA1c $(\%)$ & 0.217 & 0.029 \\
HOMA-IR & 0.187 & 0.038 \\
FINS & -0.023 & 0.422 \\
SBP $(\mathrm{mm} \mathrm{Hg})$ & 0.046 & 0.327 \\
DBP $(\mathrm{mm} \mathrm{Hg})$ & -0.028 & 0.711 \\
Cr & 0.007 & 0.883 \\
MET & 0.083 & 0.274 \\
hsCRP $(\mathrm{pg} / \mathrm{mL})$ & 0.122 & 0.027 \\
ALAT $(\mathrm{U} / \mathrm{L})$ & 0.183 & 0.002 \\
ASAT $(\mathrm{U} / \mathrm{L})$ & 0.114 & 0.037 \\
GGT $(\mathrm{U} / \mathrm{L})$ & 0.221 & $<0.001$ \\
BMI $\left(\mathrm{kg} / \mathrm{m}^{2}\right)$ & 0.133 & 0.008 \\
WC $(\mathrm{cm})$ & 0.097 & 0.039 \\
WHR & 0.144 & $<0.001$ \\
TC $(\mathrm{mmol} / \mathrm{L})$ & 0.029 & 0.149 \\
HDL-C $(\mathrm{mmol} / \mathrm{L})$ & -0.059 & 0.271 \\
LDL-C $(\mathrm{mmol} / \mathrm{L})$ & 0.227 & 0.016 \\
TG $(\mathrm{mmol} / \mathrm{L})$ & 0.221 & 0.029 \\
\hline
\end{tabular}

Correlation between variables was analysed by partial correlation test; age was adjusted.

2-hPG, 2 hour postchallenge plasma glucose; ALAT, alanine aminotransferase; ASAT, aspartate aminotransferase; BMI, body mass index; $\mathrm{Cr}$, creatinine; DBP, diastolic blood pressure; FINS, fasting serum insulin; FPG, fasting plasma glucose; GGT, $\gamma$-glutamyl transpeptidase; HbA1c, glycated haemoglobin; HDL-C, high-density lipoprotein cholesterol; HOMA-IR, homoeostasis model assessment for insulin resistance; hsCRP, high-sensitive $C$ reactive protein; LDL-C, low-density lipoprotein cholesterol; MET, metabolic equivalent; $r$, partial correlation coefficient; SBP, systolic blood pressure; TC, total cholesterol; TG, triglyceride; WC, waist circumference; WHR, waist-to-hip ratio.

logistic regression models were used to analyse the ORs and $95 \%$ CIs between the tertiles of sitting time and NAFLD. NAFLD was set as the dependent variable, and the covariates were gradually included. After adjusting for the basic model and the glycolipid metabolic biomarkers, we found that a longer sitting time ( $>7.1$ hours/day) was associated with a higher prevalence of NAFLD (OR 1.09; 95\% CI (1.04 to 1.67)).
However, this association remained insignificant after additionally adjusting for hsCRP (OR 1.03; 95\% CI (0.92 to 1.84)) (table 4).

\section{DISCUSSION}

The purpose of this study was to investigate the association between sitting time and NAFLD. We found that a longer sitting time ( $>7.1$ hours/day) was correlated with a higher prevalence of NAFLD in the Chinese male population independent of physical activity. hsCRP significantly affected this association after adjusting for confounders.

Several studies have reported the independent detrimental influence of sedentary behaviour on the development and progression of T2DM, obesity and chronic low-grade inflammation. ${ }^{10}{ }^{11}$ Frequent breaks in sedentary time improved those profiles. ${ }^{24-26}$ To the best of our knowledge, there were only three studies that reported the association between sitting time and NAFLD in humans. Hallsworth et $a l^{15}$ revealed that people with NAFLD spent more time being sedentary. Ryu et $a l^{17}$ observed a positive relationship between sitting time and NAFLD; this relationship was independent of physical activity. In a 10-year longitudinal study conducted by Helajärvi et al, ${ }^{16}$ they found that mean GGT concentrations, mean FLI and the risk of fatty liver were increased significantly in the constantly high TV time group than in the constantly low TV time groups. In agreement with those studies, we demonstrated a positive association between a longer sitting time $(>7.1$ hours/day) and the prevalence of NAFLD in a Chinese male population. Compared with the research conducted by Ryu et al, ${ }^{17}$ we also observed a positive association between sitting time and insulin resistance, while HDL-C was negatively associated with sitting time. However, we did not find any association between sitting time and SBP, DBP, HDL-C, TC, LDL-C and caloric intake based on linear regression analyses.

At present, the underlying mechanism by which sitting time contributes to the prevalence of NAFLD is unclear. First, a previous study reported that a prolonged sitting time might be involved with a higher caloric intake. ${ }^{27}$ However, no correlation was found between sitting time and caloric intake in our study. Second, it was also presumed that longer sitting may lead to lower energy consumption, which is required for active muscle contractions, causing weight gain and obesity. ${ }^{28}$ Although Ryu et $a l^{17}$ indicated that the associations between sedentary time and NAFLD may be mediated by decreased skeletal muscle mass and increased fat mass (\%), but the separate contributions of skeletal muscle and fat masses are unclear due to their collinear relationship. In this study, we did not measure skeletal muscle mass or the per cent of fat mass; although we found that BMI was an independent risk factor for sitting time, we cannot draw any conclusions about the potential role of skeletal muscle mass or the per cent fat 
Table 4 ORs $(95 \% \mathrm{Cl})$ of NAFLD according to the increased sitting time

\begin{tabular}{|c|c|c|c|}
\hline \multirow[b]{2}{*}{ Adjusted Models } & \multicolumn{3}{|c|}{ Tertiles of sitting time (hours/day) } \\
\hline & $2.2-5.0$ & $5.0-6.9$ & $>7.1$ \\
\hline *Basic model & 1 & 1.63 (1.29 to 2.85$)$ & $1.84(1.42$ to 3.17$)$ \\
\hline + Lipid profiles & 1 & 1.27 (1.06 to 2.51$)$ & 1.63 (1.31 to 2.69$)$ \\
\hline + Lipid profiles, MET & 1 & $1.42(1.21$ to 3.27$)$ & 1.42 (1.22 to 2.18$)$ \\
\hline + Lipid profiles, MET, HOMA-IR & 1 & $1.33(0.69$ to 2.15$)$ & 1.21 (1.13 to 2.05$)$ \\
\hline + Lipid profiles, MET, HOMA-IR, diabetes & 1 & $1.28(0.75$ to 1.94$)$ & 1.09 (1.04 to 1.67$)$ \\
\hline + Lipid profiles, MET, HOMA-IR, diabetes, hsCRP & 1 & 1.05 (0.66 to 1.59$)$ & $1.03(0.92$ to 1.84$)$ \\
\hline
\end{tabular}

mass in the association of sitting time and NAFLD. Third, sedentary status is considered to be linked to chronic low-grade inflammation, independent of physical activity. ${ }^{10}{ }^{11}$ Several studies revealed that systemic inflammation and mitochondrial dysfunction were implicated in the pathogenesis under sedentary conditions. ${ }^{29-}$ ${ }^{31}$ Besides, chronic inflammation is also one of the most important factors implicated in the NAFLD pathogenesis according to the 'two-hit' hypothesis. ${ }^{32}$ In animal models, it was reported that the protective effects of daily activity on plasma inflammatory indicators such as leptin, monocyte chemoattractant protein-1 (MCP-1) and tumour necrosis factor- $\alpha(\mathrm{TNF}-\alpha)$, linked to the progression of NAFLD, were either partially or completely abrogated by a transition to inactivity. ${ }^{33}$ In the hyperphagic model of Otsuka Long-Evans Tokushima fatty (OLETF) rats, Linden $e t a \hat{l}^{3}$ found that physical inactivity was associated with the development and progression of NAFLD, and the group with 16 weeks of sedentary status showed higher levels of MCP-1 and TNF- $\alpha$ compared with the group which had access to running wheels for 12 weeks which was followed by access to locking wheels for 4 weeks. ${ }^{33}$ In this study, we measured the concentrations of serum hsCRP and found that hsCRP was an independent risk factor for prolonged sitting ( $>7.1$ hours/day); this result was supported by the findings of León-Latre $e t a l .{ }^{34}$ Besides, we found that the association between longer sitting time and NAFLD disappeared after adding hsCRP to the regression model. hsCRP is an acute phase protein produced in the liver in response to inflammatory processes. ${ }^{35}$ Increasing evidence has revealed that circulating hsCRP may exist as an independent risk factor for NAFLD, and its levels are related to the severity of fibrosis. ${ }^{36} 37$ hsCRP could increase in hepatocytes under the condition of steatohepatitis compared with simple steatosis. ${ }^{38}$ Collectively, although the causal relationship between sitting time and inflammatory factors is inconclusive, we can at least hypothesise that sitting time is related to inflammatory factors, which affect NAFLD.

Certain limitations should be considered when interpreting the results of this study. First, this study was a cross-sectional observation targeted mainly at male workers, so the causal association among sitting time, NAFLD and inflammation cannot be concluded. Longitudinal studies should be conducted and gender difference should be considered in future. Second, the measures of time spent on physical activity and sedentary behaviour were obtained from a self-reported questionnaire which could be inaccurate; thus, more accurate methods should be adopted in a future study. Third, in this study, we used FLI to predict fatty liver. Although FLI has an acceptable accuracy in estimating the presence of steatosis, it cannot quantify the amount or extent of steatosis. ${ }^{19}$ Although ultrasonography and magnetic resonance imaging (MRI) of liver are considered good methods for diagnosing NAFLD, the threshold of detecting hepatic fat via ultrasound is above $30 \%,{ }^{19}$ while the cost and availability of MRI severely limit its use in clinical practice. Besides, liver biopsy is the gold standard to diagnose NAFLD, but it is unsuitable for use in epidemiological studies for ethical reasons. Also, the number of patients with NAFLD diagnosed using FLI was relatively small. These situations may have led to an underestimation, but not a reversal, of the association between sitting time and NAFLD. More longitudinal and rigorously controlled experimental studies are required to determine the chronic effects of sedentary time on NAFLD and to clarify its underlying mechanism.

\section{CONCLUSIONS}

We provided preliminary evidence that longer sitting time was positively associated with NAFLD and that this association may be affected by inflammatory indicators. Interventions on sitting time may provide a new solution for the prevention and treatment of NAFLD.

Acknowledgements The authors acknowledge American Journal Experts (Certificate Verification Key: 6D02-D622-D3D2-552A-CE85) for revising the language.

Contributors $\mathrm{HWe}, \mathrm{HQ}$ and $\mathrm{HD}$ conceived and designed the experiments. $\mathrm{HQ}$, HWa and HWe performed the experiments. HWe analysed the data and contributed to the writing of the manuscript. 
Funding This study was supported by research grants from the National Key Clinical Specialties Construction Program of China and the grants from the Chinese Society of Endocrinology and the National Natural Science Foundation of China (grant numbers 81270911, 81070639, 30771038 and 30570744).

Competing interests None declared.

Patient consent Obtained.

Ethics approval The Ethical Committees of Shanghai Jiaotong University, Shanghai, China and the first affiliated hospital of Chongqing Medical University, Chongqing, China.

Provenance and peer review Not commissioned; externally peer reviewed.

Data sharing statement No additional data are available.

Open Access This is an Open Access article distributed in accordance with the Creative Commons Attribution Non Commercial (CC BY-NC 4.0) license, which permits others to distribute, remix, adapt, build upon this work noncommercially, and license their derivative works on different terms, provided the original work is properly cited and the use is non-commercial. See: http:// creativecommons.org/licenses/by-nc/4.0/

\section{REFERENCES}

1. Cohen JC, Horton JD, Hobbs $\mathrm{HH}$. Human fatty liver disease: old questions and new insights. Science 2011;332:1519-23.

2. Goh GB, McCullough AJ. Natural History of Nonalcoholic Fatty Liver Disease. Dig Dis Sci 2016;61:1226-33.

3. Ray K. NAFLD-the next global epidemic. Nat Rev Gastroenterol Hepatol 2013;10:621

4. Fan JG, Farrell GC. Epidemiology of non-alcoholic fatty liver disease in China. J Hepatol 2009;50:204-10.

5. Zhang $\mathrm{Y}$, Zhang $\mathrm{T}$, Zhang $\mathrm{C}$, et al. Identification of reciprocal causality between non-alcoholic fatty liver disease and metabolic syndrome by a simplified Bayesian network in a Chinese population. BMJ Open 2015;5:e008204.

6. Ong JP, Pitts A, Younossi ZM. Increased overall mortality and liver related mortality in non-alcoholic fatty liver disease. $J$ Hepatol 2008;49:608-12.

7. Lonardo A, Bellentani S, Argo CK, et al. Non-alcoholic Fatty Liver Disease Study Group. Epidemiological modifiers of non-alcoholic fatty liver disease: focus on high-risk groups. Dig Liver Dis 2015;47:997-1006.

8. Ballestri S, Zona S, Targher G, et al. Nonalcoholic fatty liver disease is associated with an almost twofold increased risk of incident type 2 diabetes and metabolic syndrome. Evidence from a systematic review and meta-analysis. J Gastroenterol Hepatol 2016;31:936-44.

9. Sedentary Behaviour Research Network. Letter to the editor: standardized use of the terms "sedentary" and "sedentary behaviors". Appl Physiol Nutr Metab 2012;37:540-2.

10. Altenburg TM, Lakerveld J, Bot SD, et al. The prospective relationship between sedentary time and cardiometabolic health in adults at increased cardiometabolic risk-the Hoorn Prevention Study. Int J Behav Nutr Phys Act 2014;11:90.

11. Yates T, Khunti K, Wilmot EG, et al. Self-reported sitting time and markers of inflammation, insulin resistance, and adiposity. Am J Prev Med 2012;42:1-7.

12. Chalasani N, Younossi Z, Lavine JE et al. American Gastroenterological Association; American Association for the Study of Liver Diseases; American College of Gastroenterology. The diagnosis and management of non-alcoholic fatty liver disease: practice guideline by the American Association for the Study of Liver Diseases, American College of Gastroenterology. Gastroenterology 2012:142:1592-609.

13. Kistler KD, Brunt EM, Clark JM, et al. Physical activity recommendations, exercise intensity, and histological severity of nonalcoholic fatty liver disease. Am J Gastroenterol 2011;106:460-8; quiz 469.

14. Rector RS, Thyfault JP. Does physical inactivity cause nonalcoholic fatty liver disease? J Appl Physiol 2011;111:1828-35.

15. Hallsworth $\mathrm{K}$, Thoma $\mathrm{C}$, Moore S, et al. Non-alcoholic fatty liver disease is associated with higher levels of objectively measured sedentary behavior and lower levels of physical activity than matched healthy controls. Frontline Gastroenterol 2015;6:44-51.

16. Helajärvi $\mathrm{H}$, Pahkala $\mathrm{K}$, Heinonen OJ, et al. Television viewing and fatty liver in early midlife. The Cardiovascular Risk in Young Finns Study. Ann Med 2015;47:519-26.
17. Ryu S, Chang $\mathrm{Y}$, Jung $\mathrm{HS}$, et al. Relationship of sitting time and physical activity with non-alcoholic fatty liver disease. J Hepatol 2015;63:1229-37.

18. Ning G, Reaction Study Group. Risk Evaluation of cancers in Chinese diabetic Individuals: a longitudinal (REACTION) study. J Diabetes 2012;4:172-3.

19. Fedchuk L, Nascimbeni F, Pais R, et al. Performance and limitations of steatosis biomarkers in patients with nonalcoholic fatty liver disease. Aliment Pharmacol Ther 2014;40:1209-22.

20. Zelber-Sagi S, Webb M, Assy N, et al. Comparison of fatty liver index with noninvasive methods for steatosis detection and quantification. World J Gastroenterol 2013;19:57-64.

21. World Health Organization. Definition, diagnosis and classification of diabetes mellitus and its complications, Report of a WHO Consultation. Part 1. Diagnosis and classification of diabetes mellitus. Geneva: Department of Noncommunicable Disease Surveillance, 1999. WHO/NCD/NCS/99.2.

22. Anuurad E, Shiwaku K, Nogi A, et al. The new BMI criteria for Asians by the regional office for the western pacific region of WHO are suitable for screening of overweight to prevent metabolic syndrome in elder Japanese workers. J Occup Health 2003;45:335-43.

23. Ainsworth BE, Haskell WL, Herrmann SD, et al. 2011 Compendium of Physical Activities: a second update of codes and MET values. Med Sci Sports Exerc 2011;43:1575-81.

24. Healy GN, Dunstan DW, Salmon J, et al. Breaks in sedentary time: beneficial associations with metabolic risk. Diabetes Care 2008;31:661-6.

25. Healy GN, Matthews CE, Dunstan DW, et al. Sedentary time and cardio-metabolic biomarkers in US adults: NHANES 2003-06. Eur Heart J 2011;32:590-7.

26. Peddie MC, Bone JL, Rehrer NJ, et al. Breaking prolonged sitting reduces postprandial glycemia in healthy, normal-weight adults: a randomized crossover trial. Am J Clin Nutr 2013;98:358-66.

27. Krishnan S, Rosenberg L, Palmer JR. Physical activity and television watching in relation to risk of type 2 diabetes, the Black Women's Health Study. Am J Epidemiol 2009;169: 428-34.

28. Hamilton MT, Hamilton DG, Zderic TW. Role of low energy expenditure and sitting in obesity, metabolic syndrome, type 2 diabetes, and cardiovascular disease. Diabetes 2007;56:2655-67.

29. Rector RS, Thyfault JP, Uptergrove GM, et al. Mitochondrial dysfunction precedes insulin resistance and hepatic steatosis and contributes to the natural history of non-alcoholic fatty liver disease in an obese rodent model. J Hepatol 2010;52:727-36.

30. Zou X, Yan C, Shi Y, et al. Mitochondrial dysfunction in obesity-associated nonalcoholic fatty liver disease: the protective effects of pomegranate with its active component punicalagin. Antioxid Redox Signal 2014;21:1557-70.

31. Hamilton MT, Hamilton DG, Zderic TW. Exercise physiology versus inactivity physiology: an essential concept for understanding lipoprotein lipase regulation. Exerc Sport Sci Rev 2004;32: 161-6.

32. Day CP, James OF. Steatohepatitis: a tale of two "hits"? Gastroenterology 1998;114:842-5.

33. Linden MA, Meers GM, Ruebel ML, et al. Hepatic steatosis development with four weeks of physical inactivity in previously active, hyperphagic OLETF rats. Am J Physiol Regul Integr Comp Physiol 2013;304:R763-71.

34. León-Latre M, Moreno-Franco B, Andrés-Esteban EM, et al. Aragon Workers' Health Study investigators. Sedentary lifestyle and its relation to cardiovascular risk factors, insulin resistance and inflammatory profile. Rev Esp Cardiol (Engl Ed) 2014;67:449-55.

35. Maleki I, Rastgar A, Hosseini V, et al. High sensitive CRP and pentraxine 3 as noninvasive biomarkers of nonalcoholic fatty liver disease. Eur Rev Med Pharmacol Sci 2014;18:1583-90.

36. Oruc N, Ozutemiz O, Yuce G, et al. Serum procalcitonin and CRP levels in non-alcoholic fatty liver disease: a case control study. BMC Gastroenterol 2009:9:16.

37. Kogiso T, Moriyoshi Y, Shimizu S, et al. High-sensitivity C-reactive protein as a serum predictor of nonalcoholic fatty liver disease based on the Akaike Information Criterion scoring system in the general Japanese population. J Gastroenterol 2009;44:313-21.

38. Yoneda $\mathrm{M}$, Mawatari $\mathrm{H}$, Fujita $\mathrm{K}$, et al. High-sensitivity C-reactive protein is an independent clinical feature of nonalcoholic steatohepatitis (NASH) and also of the severity of fibrosis in NASH. J Gastroenterol 2007;42:573-82. 